\title{
Outcome of stapled haemorrhoidopexy versus doppler-guided haemorrhoidal artery ligation for grade III haemorrhoids
}

\author{
P. Giordano
}

Received: 5 July 2011/Accepted: 7 July 2011/Published online: 29 July 2011

(C) Springer-Verlag 2011

Currently, multiple operations are available for the treatment of symptomatic haemorrhoids, and no method is universally accepted as superior to the others. The operative approach is often tailored to the individual patient depending on the surgeon's preference and experience. Ideally, the surgical procedure for the treatment of haemorrhoids should be a technique able to provide long-term relief of symptoms whilst being economical, safe, easy to perform and well tolerated by patients. In recent years, we have seen a shift towards less invasive procedures mostly aimed at avoiding or minimizing postoperative pain. New techniques such as stapled haemorrhoidopexy (SH) and doppler-guided haemorrhoidal artery ligation (DGHAL) also known as transanal haemorrhoidal artery ligation (THD) have now become widely accepted for the treatment of symptomatic haemorrhoids. Both SH and DGHAL have the advantage of not leaving wounds in the anal canal which improves convalescence by reducing postoperative discomfort. The expected reduction in postoperative pain makes these procedures appealing to the patients, and indeed, their short-term benefits compared to conventional surgery have been clearly demonstrated in prospective comparative trials and/or observational studies [1-3]. However, the real innovation introduced by these procedures is the fact that they aim to treat the symptoms whilst not excising the diseased tissue, as in conventional haemorrhoidectomy $(\mathrm{CH})$, but by restoring the normal anatomy and correcting the physiology of the haemorrhoidal plexus. This means that the long-term benefits are potentially by far more important than the short-term ones. With that in

P. Giordano $(\bowtie)$

Department of Colorectal Surgery, Whipps Cross University

Hospital, Whipps Cross Road, London E11 1NR, UK

e-mail: pasquale.giordano@whippsx.nhs.uk mind, the question is whether they actually work and whether any of these new modalities of treatment is superior to the other. In this issue of techniques in coloproctology, Avital et al. [4] showed that pain is significantly lower in the DGHAL group during the first $24 \mathrm{~h}$, at the time of the first bowel movement, and at 1 week after the operation. However, in this study, a mucopexy in association with the DGHAL was never performed. It could be argued that performing a plication of the rectal mucosa may improve outcomes but may also lead to more postoperative pain. Two different prospective trials comparing SH to THD with rectal mucopexy as standard have shown less pain and earlier recovery following the THD procedure [5, 6]. However, reduced postoperative pain although important is not a measure of success for which safety and effectiveness remain the main benchmarks. To date, there are only a very few other studies comparing the two techniques directly and only one reporting results at a relatively long follow-up. Based on the evidence available from comparative studies, these procedures have demonstrated to be equally effective. In Avital et al.'s [4] study, $18 \%$ of patients in the DGHAL group suffered from ongoing haemorrhoidal symptoms whilst only $3 \%$ of the patients in the $\mathrm{SH}$ group did. This reflected in a higher number of patients requiring further intervention in the DGHAL group. However, once again it could be argued that the patients in the DGHAL group did not receive any mucopexy and this may have certainly influenced the outcome. Indeed, in the prospective trials where rectal mucopexy was used as a standard part of the THD procedure, the improvement of symptoms was similar in both groups $[5,6]$. In the few comparative studies available, one major complication was reported following $\mathrm{SH}$ whilst none were encountered in the DGHAL patients [6]. Outside these studies, a significant number of major complications 
have been described following SH [7]. On the contrary, no major problems have ever been reported following DGHAL/THD. It is unlikely that this difference can be explained by the fact that SH gained popularity earlier than DGHAL, and the literature on SH is by far more extensive than that on the other technique. Giving a definite explanation of why major complications may occur following $\mathrm{SH}$ is impossible; however, it is possible that the "blind" excision of part of the rectal wall performed with this technique is somehow related to the complications. The fact that THD is a non-excisional technique should virtually eliminate the possibility of major problems being associated with it.

A recent meta-analysis of long-term outcomes of SH versus $\mathrm{CH}$ demonstrated a significantly higher overall rate of prolapse recurrence in the SH group [8]. The difference was even more significant in the subset of patients with III and IV degree haemorrhoids. We currently do not have enough data on long-term outcomes for DGHAL/THD and therefore we do not know at this stage whether the encouraging early results obtained using this technique will be maintained over time. Large multicentre trials are expected to better define the role of these new procedures.

There is no doubt that SH and DGHAL/THD have been able to capture the interest of surgeons; we now have to see whether they will able to stand the test of time.

Conflict of interest Mr. Pasquale Giordano is a trainer in the THD technique.

\section{References}

1. Nisar PJ, Acheson AG, Neal KR, Scholefield JH (2004) Stapled hemorrhoidopexy compared with conventional hemorrhoidectomy: systematic review of randomized, controlled trials. Dis Colon Rectum 47:1837-1845

2. Bursics A, Morvay K, Kupcsulik P, Flautner L (2004) Comparison of early and 1-year follow-up results of conventional hemorrhoidectomy and hemorrhoid artery ligation: a randomized study. Int $\mathrm{J}$ Colorectal Dis 19:176-180

3. Khafagy W, El Nakeeb A, Fouda E et al (2009) Conventional haemorrhoidectomy, stapled haemorrhoidectomy, doppler guided haemorrhoidectomy artery ligation; post operative pain and anorectal manometric assessment. Hepatogastroenterology 56:1010-1015

4. Avital S, Itah R, Skornick Y, Greenberg R (2011) Outcome of stapled hemorrhoidopexy versus doppler-guided hemorrhoidal artery ligation for grade III haemorrhoids. Tech Coloproctol 15 . doi:10.1007/s10151-011-0699-z

5. Festen S, van Hoogstraten MJ, van Geloven AA, Gerhards MF (2009) Treatment of grade III and IV haemorrhoidal disease with PPH or THD. A randomised trial on postoperative complication and short-term results. Int J Colorectal Dis 24:1401-1405

6. Giordano P, Nastro P, Davies A, Gravante G (2011) Prospective evaluation of stapled haemorrhoidopexy versus transanal haemorrhoidal dearterialisation for stage II and III haemorrhoids: 3-year outcomes. Tech Coloproctol 15:67-73

7. Pescatori M, Gagliardi G (2008) Postoperative complications after procedure for prolapsed hemorrhoids (PPH) and stapled transanal rectal resection (STARR) procedures. Tech Coloproctol 12:7-19

8. Giordano P, Gravante G, Sorge R, Ovens L, Nastro P (2009) Longterm outcomes of stapled hemorrhoidopexy versus conventional hemorrhoidectomy: a meta-analysis of randomized controlled trials. Arch Surg 144:266-272 